\title{
Multiple myeloma coexisting colon carcinoma as the presenting symptom of a neutrophilic leukemoid reaction: a rare case report with genetic analysis
}

\section{Manxiong Cao}

The First Affiliated Hospital of Shantou University Medical college

\section{Zhanqin Huang}

Shantou University Medical College

Huanbing Zhou

The first Affiliated Hospital of Shantou university medical college

Dongqing Zhang ( $\square$ zhangdongqing1976@163.com )

The First Affiliated Hospital of Shantou University Medical College https://orcid.org/0000-0002-20633994

\section{Case Report}

Keywords: Leukemoid reaction, Chronic neutrophilic leukemia, Multiple myeloma, Colon carcinoma

Posted Date: December 28th, 2020

DOl: https://doi.org/10.21203/rs.3.rs-135225/v1

License: (c) (i) This work is licensed under a Creative Commons Attribution 4.0 International License. Read Full License 


\section{Abstract}

\section{Background}

Leukemoid reaction refers to reactive leukocytosis exceeding 50,000 cells/ $\mu$ l. Chronic neutrophilic leukemia is a rare clonal hematopoietic disorder characterized by sustained mature neutrophilia in the absence of monocytosis or basophilia. The differentiation between leukemoid reaction and chronic neutrophilic leukemia is problematic because both conditions share similar morphological features.

Case presentation

Here, we present an extremely rare case of a 62-year-old male patient who was initially diagnosed with chronic neutrophilic leukemia at another hospital. When the patient came to our hospital, no mutations in the CSF3R, SETBP1, ASXL1, TET2, SRSF2, SF3B1, ZRSR2 and U2AF1F genes were found by whole-exon sequencing. Further examination revealed the presence of immunoglobulin $\mathrm{G}$ kappa myeloma. Meanwhile, colonoscopy showed a mass in the colon, and biopsy confirmed the presence of colon adenocarcinoma. Therefore, we suggest that the increased white blood cell count in the patient was merely a neutrophilic leukemoid reaction caused by synchronous multiple myeloma and colon carcinoma.

\section{Conclusion}

This is the first report of a case of coexisting multiple myeloma and colon carcinoma presenting with a neutrophilic leukemoid reaction. This case was presented to illustrate that the diagnosis of chronic neutrophilic leukemia must meet the strict WHO diagnostic criteria, especially if there is an underlying plasma cell disorder, and myeloid clonality must be demonstrated to make a distinction between chronic neutrophilic leukemia and leukemoid reaction. Meanwhile, this case provides us with a reference that further examination, especially pathological examination, should be performed on a patient diagnosed with multiple myeloma, who has extramedullary lesions, because other primary tumors may be also presenting the very similar symptoms. Pathological examination is helpful to differentiate the primary tumor from extramedullary invasion of multiple myeloma.

\section{Background}

Leukemoid reaction (LR) is a hematologic finding defined as a leukocyte count above $50,000 \mathrm{cells} / \mu \mathrm{\mu}$. It is characterized by an increase in mature neutrophils and a marked "left shift", which refers to the presence of immature granulocytic forms (1). The main causes of LR are severe infection, neoplasms, toxin exposure, severe hemorrhage and acute hemolysis (2-4). Cancer is an important cause of LR, which can be identified at either diagnosis or prior to it by as much as 4 years (5).

Chronic neutrophilic leukemia $(\mathrm{CNL})$ is a rare clonal hematopoietic disorder characterized by sustained mature neutrophilia in the absence of monocytosis or basophilia (6). LR and CNL are closely resemble 
one another and frequently pose a diagnostic dilemma, particularly when the leukocyte count is very high (7). In this report, we describe a case of a patient who presented with a highly significant increase in the leukocyte count and was diagnosed with CNL at another hospital. However, when he was transferred to our hospital, he was diagnosed with a neutrophilic LR caused by synchronous multiple myeloma (MM) and colon carcinoma.

\section{Case Presentation}

The patient was a 62-year-old Chinese man who was admitted to another hospital on October 9, 2019, due to abdominal distension and intermittent left lower abdominal pain for one week. Physical examination revealed abdominal distension and spleen rib under $6 \mathrm{~cm}$. Hematological investigations showed a significantly increased white blood cell count and moderate anemia (white blood cell count, $133.82 \times 10^{9} /$; red blood cell count, $2.30 \times 10^{12} /$; hemoglobin level, $76 \mathrm{~g} / \mathrm{l}$ and platelet count, $105 \times$ $\left.10^{9} / \mathrm{I}\right)$. Biochemical tests indicated a marked elevation of globulin. Monoclonal bands were found by serum immunofixation electrophoresis. Abdominal ultrasonography revealed a large spleen. Molecular genetic studies were performed to screen BCR/ABL 1, PDGFRA, PDGFRB, and FGFR1 rearrangements and point mutations in CSF3R, JAK2, CALR, and MPL, and the results were all negative. Bone marrow plasma cells accounted for $2 \%$. The patient was diagnosed with "CNL and monoclonal gammopathy of undetermined significance (MGUS)" at this hospital and treated with splenectomy and hydroxyurea. After these treatments, the patient's abdominal distention was reduced, and the white blood cell count dropped to $32.21 \times 10^{9} / \mathrm{l}$. On December 6,2019 , the patient was admitted to the emergency intensive care unit (ICU) of our hospital due to cough for more than half a month, aggravation with shortness of breath for one week, and confusion for 7 hours. A chest radiograph showed bilateral pneumonia. His condition improved after endotracheal intubation and anti-infective treatment. And then he was transferred to the hematology department for further diagnosis and treatment.

Routine blood tests revealed a hemoglobin level of $47 \mathrm{~g} / \mathrm{l}$ (normal range, $130-175 \mathrm{~g} / \mathrm{l}$ ), a red blood cell count of $1.02 \times 10^{12} /$ I (normal range, 4.30-5.80 $\times 10^{12} / \mathrm{l}$ ), a white blood cell count of $75.76 \times 10^{9} / \mathrm{I}$ (normal range, 3.50-9.50 $\times 10^{9} / \mathrm{I}$ ) and a platelet count of $70 \times 10^{9} / \mathrm{I}$ (normal range, $\left.150-350 \times 10^{9} / \mathrm{I}\right)$. A peripheral blood film ( $1 \%$ neutrophilic myelocytes, $2 \%$ neutrophilic metamyelocytes, $65 \%$ band-stage neutrophils, $26 \%$ segmented neutrophils, $3 \%$ lymphocytes, and $3 \%$ monocytes) indicated leukocytosis with an increased number of segmented and band-stage neutrophils with clear cytoplasmic toxic granules and Döhle bodies. A small number of myelocytes and an occasional blast were noted; however, basophilia or eosinophilia were not observed (Fig. 1A). Furthermore, the patient's peripheral blood neutrophil alkaline phosphatase (NAP) score was 293 (normal range, 40-80).

Bone marrow aspiration demonstrated notably hypercellular marrow with marked granulocytic proliferation, predominantly consisting of band-stage and segmented neutrophils, with clear cytoplasmic toxic granules and Döhle bodies. Erythroid and megakaryocytic cell levels were decreased. However, the morphology was normal. In addition, the myeloid:erythroid ratio was 16.7:1, and there was no increase in 
the proportion of basophils and eosinophils (Fig. 1B). A trephine biopsy of the bone marrow demonstrated similar results, and there was no increase in the number of reticulin fibers. Flow cytometry analysis was consistent with marked myeloid hyperplasia without an increase in the number of blasts or monocytes. However, it was difficult to distinguish CNL from LR based on the above findings. The diagnosis should be confirmed by demonstrating neutrophil clonality. Therefore, cytogenetic and molecular studies were conducted on the patient.

Conventional cytogenetic analysis revealed a normal karyotype [46, XY (20 cells)] and an absence of the Philadelphia $(\mathrm{Ph})$ chromosome. Furthermore, molecular biology investigations demonstrated no mutations in the CSF3R gene, negativity for p210 BCR/ABL 1, p230 BCR/ABL 1 and $p 190 B C R / A B L 1$ fusion proteins, an absence of the JAK2V617F mutation, and no mutations in PDGFRA/PDGFRB. Furthermore, using whole-exon sequencing (WES), we did not find any mutations in CSF3R, SETBP1, ASXL1, TET2, SRSF2, SF3B1, ZRSR2, or U2AF1. Therefore, the diagnosis of CNL could not be established. Meanwhile, it is necessary to find out the reason and the explanation for the significantly increased neutrophils counting of the patient.

The biochemical examination showed that the creatinine level was $147.04 \mu \mathrm{mol} / \mathrm{I}$ (normal range, 45$133 \mu \mathrm{mol} / \mathrm{l}$ ) and uric acid was $567 \mu \mathrm{mol} / \mathrm{I}$ (normal range, 238-506 $\mu \mathrm{mol} / \mathrm{l}$ ). The serum albumin and globulin levels were $21.43 \mathrm{~g} / \mathrm{l}$ (normal range, 40.0-55.0 g/l) and $65.34 \mathrm{~g} / \mathrm{l}$ (normal range, 20-40 g/l), respectively, indicating a reversal of the albumin/globulin ratio (0.33; normal range, 1.2-2.4). Serum immunoglobulin analysis revealed immunoglobulin $\mathrm{G}(\mathrm{IgG})$ at $55.7 \mathrm{~g} / \mathrm{l}$ (reference values: $7.51-15.6 \mathrm{~g} / \mathrm{l}$ ), $\lg A$ at $0.46 \mathrm{~g} / \mathrm{l}$ (reference values: $0.82-4.53 \mathrm{~g} / \mathrm{l}$ ), and $\mathrm{IgM}$ at $0.26 \mathrm{~g} / \mathrm{l}$ (reference values: $0.46-3.04 \mathrm{~g} / \mathrm{l}$ ). Serum protein electrophoresis was positive for a monoclonal spike (M-spike) of $67.2 \%$ in the beta region. Serum immunofixation electrophoresis indicated the presence of monoclonal bands in the $\lg \mathrm{G}$ and kappa lanes. A computed tomography (CT) scan revealed an osteolytic lesion in the left ilium. Based on these findings, MM was highly suspected. Therefore, bone marrow aspiration and biopsy were performed for the pathological diagnosis. Bone marrow aspiration from the right posterior iliac revealed only a mild increase in plasma cells (2.5\% plasma cells of the ANC) (Fig. 1B), but aspiration from the left posterior iliac revealed a moderate increase in plasma cells (18.5\% plasma cells of the ANC) (Fig. 1C), and clusters of distributed plasma cells (Fig. 1D) could be easily seen. Bone marrow biopsy revealed markedly hypercellular marrow and clusters of distributed plasma cells. Immunohistochemical staining from left posterior iliac bone was positive for CD38, CD138 and CD56. Kappa and lambda light chain in situ hybridization showed the monoclonal expression of the kappa light chain, which was in accordance with plasmacytoma (Fig. 2). Flow cytometry of bone marrow aspirates revealed that the proportion of atypical cells was $8.6 \%$. These cells exclusively expressed kappa chains, showed strong CD38 and CD138 expression and simultaneously expressed CD117 and CD56. All these findings led to the diagnosis of MM.

The patient had intermittent pain in the left lower abdomen during the course of the disease. Abdominal CT showed marked thickening of the middle intestinal wall of the left descending colon and narrowing of the intestine (Fig. 3A). At first, MM was suspected to have extramedullary invasion. However, Tumor 
markers, including CEA (340.47 ng/ml; normal range, 0.15-9.7 ng/ml), CA199 (797.80 U/ml; normal range, $<35.00 \mathrm{U} / \mathrm{ml}$ ) were significantly elevated. Colonoscopy showed a cauliflower mass in the colon (Fig. 3B), and biopsy showed adenocarcinoma of the colon without plasma cell involvement (Fig. 3C). At this point, the diagnosis of a primary colon tumor was clear.

\section{Discussion}

As defined in the 2016 World Health Organization (WHO) classification, CNL is a Philadelphia-negative myeloproliferative neoplasm (MPN) characterized by peripheral blood leukocytosis of $\geq 25 \times 10^{9} /$ I (of which $>80 \%$ are segmented neutrophils plus band forms and $<10 \%$ are neutrophil precursors with rare myeloblasts), monocyte counts $<1 \times 10^{9} / \mathrm{I}$ and the absence of dysgranulopoiesis, as well as hypercellular bone marrow with granulocyte hyperplasia, normal maturation and $<5 \%$ myeloblasts. The remaining components are exclusionary and include the absence of the fulfillment of the WHO criteria for another MPN and the absence of rearrangements in PDGFRA, PDGFRB, FGRF1, or PCM1-JAK2. The WHO endorsed the presence of CSF3RT618/ or other activating CSF3R mutations as a key diagnostic criterion for CNL. The WHO also accounted for CSF3R-negative disease in the face of chronic neutrophilia (minimum of 3 months), splenomegaly, and the exclusion of reactive leukocytosis. If there is an underlying plasma cell disorder, myeloid clonality must be demonstrated to make the additional diagnosis of CNL (8). Though the absence of a CSF3R mutation does not exclude the possibility of CNL, it should prompt a careful review of the diagnosis. Starting in the early 1900s, more than 200 cases of CNL have been described, but the true occurrence of CNL is likely much lower when the strict WHO diagnostic criteria are applied. This has recently been detailed in a review by Bain and Ahmad, which identified a total of 52 published cases of "CNL" that did not meet the WHO diagnostic criteria for the disease. Recent evidence suggests that the great majority of such cases represent a neutrophilic LR to underlying MM or MGUS (7). Distinguishing CNL from a LR may be challenging, as both may present with significant neutrophilia, bone marrow hypercellularity, normal cytogenetics, and the absence of the $B C R$ $A B L 1$ fusion gene. White blood cell counts may be more modestly elevated in LR, though there have been reports of white blood cell counts up to $100 \times 10^{9} / \mathrm{I}(9,10)$. Neutrophil toxic granulations and Döhle bodies are a common finding in CNL and may be suggestive of an activated neutrophil state. Notably, these same findings are more frequently observed in neutrophilic LRs but remain nonspecific (7). The demonstration of clonality, including the identification of a CSF3R mutation or other molecular or cytogenetic abnormalities, is clearly valuable for a diagnosis in favor of CNL (11). In this case, the patient was initially diagnosed with CNL at another hospital. He had a large spleen and a significantly increased white blood cell count with clear toxic granulation, Döhle bodies and a significantly increased NAP score. All of these changes are not valuable for making a distinction. The diagnosis should be confirmed by demonstrating neutrophil clonality. Therefore, when the patient came to our hospital, we performed cytogenetic and molecular studies on the patient, and the results showed a normal karyotype and no mutations in CSF3R, SETBP1, ASXL1, TET2, SRSF2, SF3B1, ZRSR2, or U2AF1. Therefore, the diagnosis of CNL could not be made according to the WHO 2016 classification. The patient was eventually diagnosed with MM and colon carcinoma at our hospital, so we believe that the patient's elevated white blood cell 
count was a neutrophilic LR caused by these two conditions. This situation is rare, likely resulting from interleukin-6 (IL-6) and granulocyte colony-stimulating factor (G-CSF) produced by neoplastic cells (1214).

MM is a plasma cell dyscrasia characterized by neoplastic proliferation of plasma cells secreting a monoclonal immunoglobulin. The diagnosis of active MM as established by the International Myeloma Working Group criteria 2 requires clonal bone marrow plasma cells $\geq 10 \%$ or biopsy-proven bony or extramedullary plasmacytoma plus the presence of either a related organ or tissue impairment (anemia, hypercalcemia, renal insufficiency, and bone lesions) or myeloma-defining events (60\% or greater clonal plasma cells on bone marrow examination, serum involved/uninvolved free light chain ratio of 100 or greater, or more than one focal lesion on magnetic resonance imaging (MRI) that is at least $5 \mathrm{~mm}$ or greater in size). Malignant plasma cells uniformly overexpress CD38, CD138, and CD56 molecules (15).

Because the bone marrow plasma cell proportion did not meet the criteria for the diagnosis of MM, the patient was initially diagnosed with MGUS at another hospital. At our hospital, the right iliac bone marrow plasma cell proportion of the patient was only $2.5 \%$, but the left iliac bone marrow plasma cell proportion was up to $18.5 \%$ and it was easy to see a naive and clumped distribution of plasma cells. CT showed an osteolytic lesion in the left ilium. Bone marrow biopsy and flow cytometry analysis were consistent with plasma cell myeloma. Thus, for patients with suspected MM, multiple sites of bone marrow aspiration, bone marrow biopsy, $\mathrm{CT}, \mathrm{MRI}$, and positron emission tomography/computed tomography (PET/CT) are recommended to improve the diagnosis rate.

It was also interesting that the clinical observation of intermittent pain in the left lower abdomen of this patient as well as the significantly thickened middle intestinal wall of the left descending colon with abdominal CT scanning suggested the extramedullary invasion in colon of multiple myeloma. However, the significantly increased tumor markers of CEA and CA199, especially the evidences of colonoscopy and pathological biopsy confirmed the diagnosis of colon adenocarcinoma. So, this case provides us with a reference that further examination, especially pathological examination, should be performed on a patient diagnosed with $\mathrm{MM}$, who has extramedullary lesions, because other primary tumors may be also presenting the very similar symptoms. Pathological examination is helpful to differentiate the primary tumor from extramedullary invasion of multiple myeloma.

\section{Conclusion}

A patient with synchronous MM and colon carcinoma presenting with LR is extremely rare. To the best of our knowledge, this situation has not been previously reported in the literatures. This case was presented to illustrate that the diagnosis of CNL must meet the strict WHO diagnostic criteria, especially if there is an underlying plasma cell disorder, and myeloid clonality must be demonstrated to make a distinction between CNL and LR.

\section{Abbreviations}


LR: Leukemoid reaction

CNL: Chronic neutrophilic leukemia

MM: multiple myeloma

MGUS: monoclonal gammopathy of undetermined significance

ICU: intensive care unit

CT: computed tomography

WES: whole-exon sequencing

WHO: World Health Organization

MPN: myeloproliferative neoplasm

NAP: neutrophil alkaline phosphatase

CEA: carcinoembryonic antigen

CA199: carbohydrate antigen 199

\section{Declarations}

\section{Availability of data and materials}

The data sets used and/or analyzed during the current study are available from the corresponding author on reasonable request.

\section{Acknowledgments}

We would like to thank AJE (www.aje.com) for English language editing.

\section{Funding}

This work is supported in part by research grants from the National Natural Science Found of China (No 30901810). Zhanqin Huang is the sponsor.

\section{Contribution}

Zhanqin Huang and Dongqing Zhang designed the study, analyzed the data, and drafted the manuscript. Manxiong Cao, Huanbing Zhou,and Dongqing Zhang were involved in collecting patient information and analyzing the data. All authors have read and approved the final manuscript. 


\section{Ethics approval and consent to participate}

This study was approved by the Ethical Committee of The First Affiliated Hospital of Shantou University Medical College.

\section{Consent for publication}

The patient provided written informed consent for publication of this report and accompanying images prior to study enrollment.

\section{Competing interests}

The authors declare that they have no competing interests.

\section{References}

1. Sakka V, Tsiodras S, Giamarellos-Bourboulis EJ, Giamarellou H. An update on the etiology and diagnostic evaluation of a leukemoid reaction. Eur J Intern Med. 2006; 17(6):394-8.

2. Granger JM, Kontoyiannis DP. Etiology and outcome of extreme leukocytosis in 758 nonhematologic cancer patients: a retrospective, single-institution study. Cancer. 2009; 115(17):3919-23.

3. Naaraayan A, Aleta M, Basak P, Jesmajian S, Goldstein R. Leukemoid reaction to Clostridium difficile infection. Anaerobe. 2015; 34:158-60.

4. Li W, Repnikova E. ALK-positive anaplastic large-cell lymphoma with marked leukemoid reaction and hemophagocytic lymphohistiocytosis. Blood. 2016; 127(16):2041.

5. Nimieri HS, Makoni SN, Madziwa FH, Nemiary DS. Leukemoid reaction response to chemotherapy and radiotherapy in a patient with cervical carcinoma. Ann Hematol. 2003; 82(5):316-7.

6. Elliott MA, Hanson CA, Dewald GW, Smoley SA, Lasho TL, Tefferi A. WHO-defined chronic neutrophilic leukemia: a long-term analysis of 12 cases and a critical review of the literature. Leukemia. 2005; 19(2):313-7.

7. Bain BJ, Ahmad S. Chronic neutrophilic leukaemia and plasma cell-related neutrophilic leukaemoid reactions. Br J Haematol. 2015; 171(3):400-10.

8. Arber DA, Orazi A, Hasserjian R, et al. The 2016 revision to the World Health Organization classification of myeloid neoplasms and acute leukemia. Blood. 2016; 127(20):2391-405.

9. Eichenhorn MS, Van Slyck EJ. Marked mature neutrophilic leukocytosis: a leukemoid variant associated with malignancy. Am J Med Sci. 1982; 284(3):32-6.

10. Thakral B, Loghavi S. Marked paraneoplastic leukemoid reaction in a patient with mesothelioma mimicking a myeloid neoplasm. Blood. 2020; 135(6):457.

11. Bohm J, Kock S, Schaefer HE, Fisch P. Evidence of clonality in chronic neutrophilic leukaemia. J Clin Pathol. 2003; 56(4):292-5. 
12. Katoh Y, Nakamura M, Ohnishi Y, Shimamura K, Ueyama Y, Tamaoki N. Autonomous production of granulocyte-colony stimulating factor in tumour xenografts associated with leukocytosis. $\mathrm{Br} \mathrm{J}$ Cancer. 1993; 68(4):715-9.

13. Jardin $F$, Vasse $M$, Debled $M$, et al. Intense paraneoplastic neutrophilic leukemoid reaction related to a G-CSF-secreting lung sarcoma. Am J Hematol. 2005; 80(3):243-5.

14. Csiszar A, Szentes T, Haraszti B, Balazs A, Petranyi GG, Pocsik E. The pattern of cytokine gene expression in human colorectal carcinoma. Pathol Oncol Res. 2004; 10(2):109-16.

15. Rajkumar SV, Dimopoulos MA, Palumbo A, et al. International Myeloma Working Group updated criteria for the diagnosis of multiple myeloma. Lancet Oncol. 2014; 15(12):e538-48.

\section{Figures}




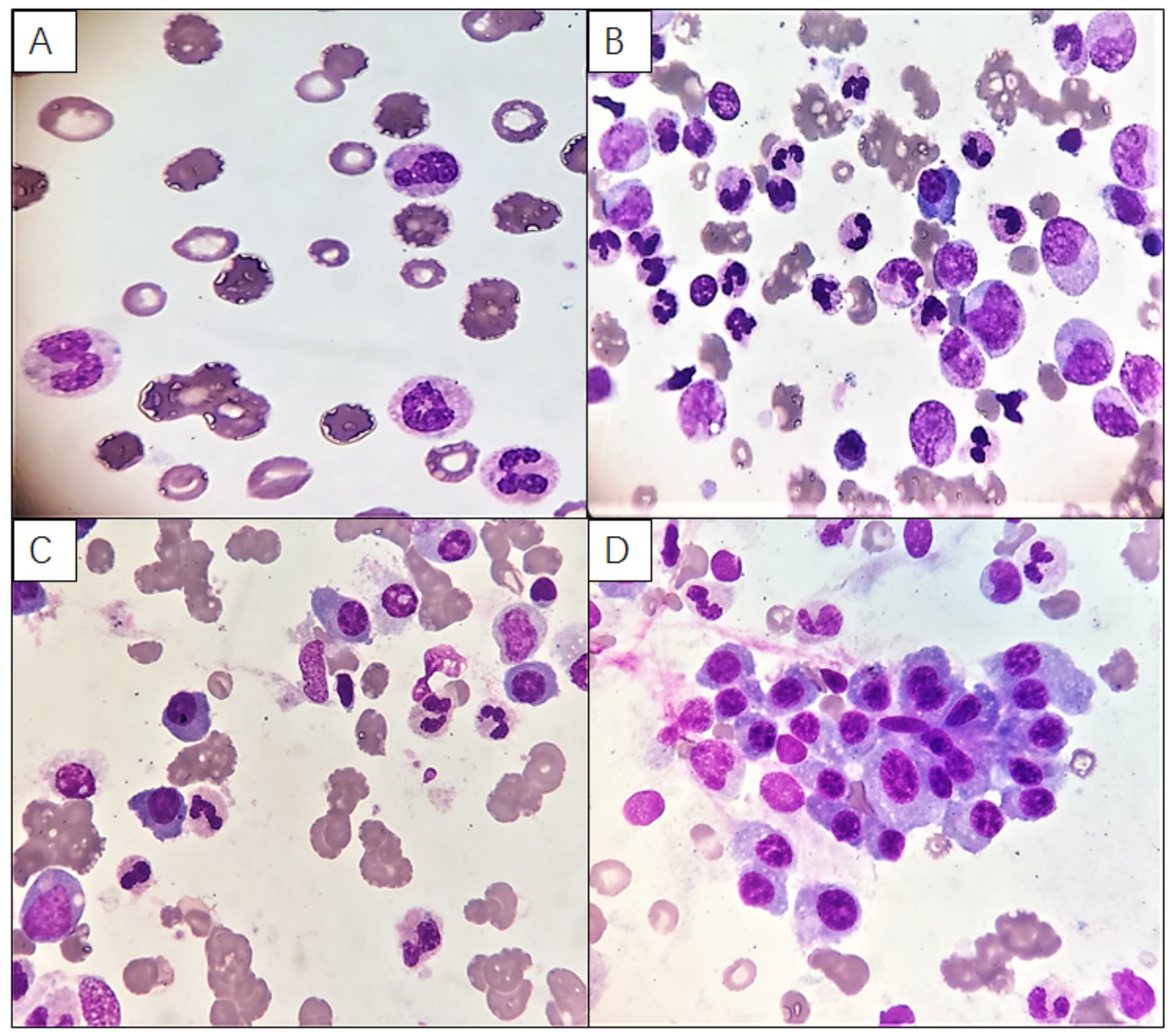

Figure 1

Peripheral blood film (A, Wright-Giemsa stain, original magnification $\times 1200)$ showing neutrophilia with toxic granules and Döhle bodies. Bone marrow aspiration from the right posterior iliac (B, Wright-Giemsa stain, original magnification $\times 1000$ ) showing marked granulocytic proliferation with a small number of plasma cells. Bone marrow aspiration from the left posterior iliac showing a moderate number of plasma cells $(C$, Wright-Giemsa stain, original magnification $\times 1000)$ and clusters of distributed plasma cells $(D$, Wright-Giemsa stain, original magnification $\times 1000)$. 


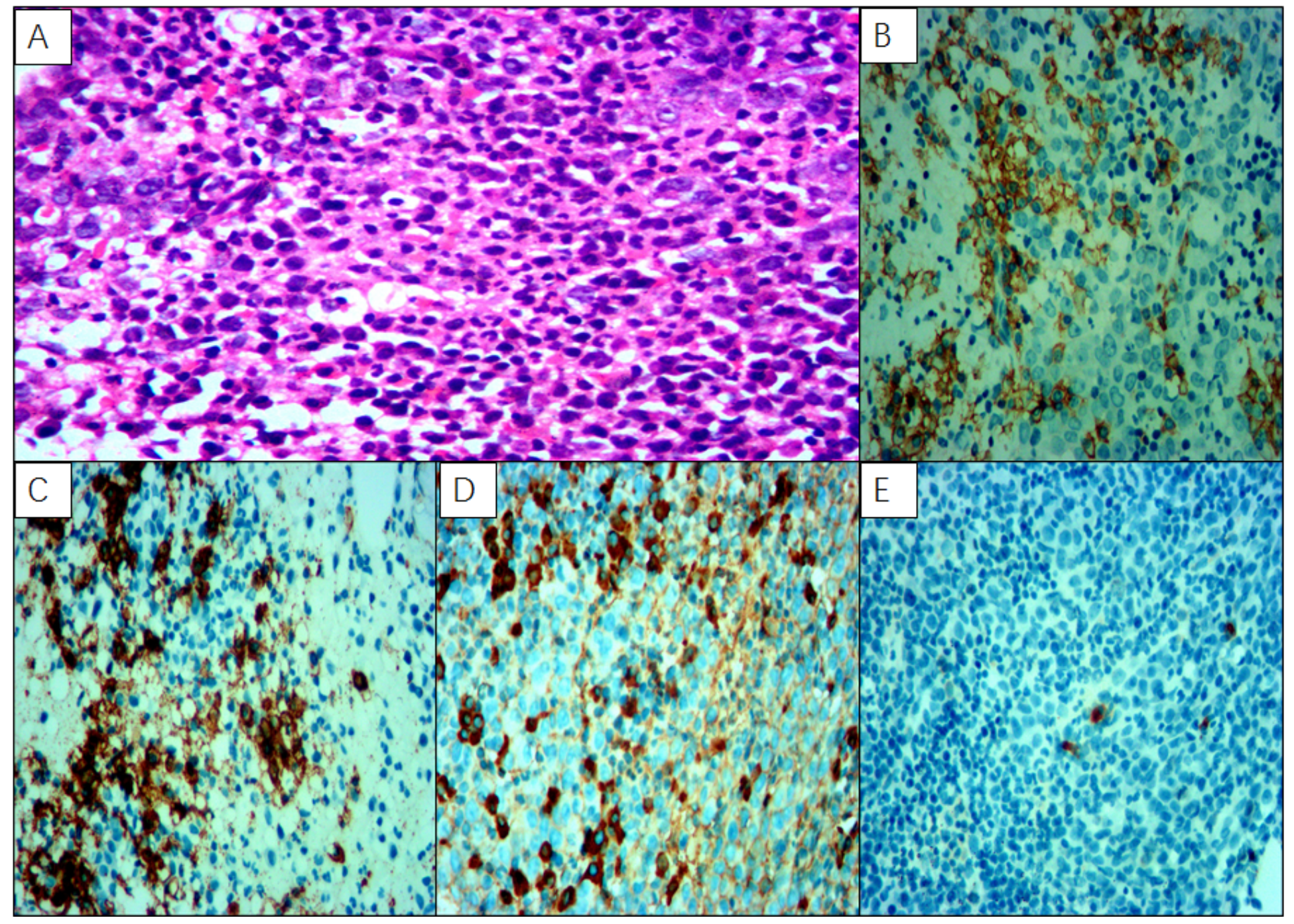

Figure 2

Bone marrow biopsy revealed markedly hypercellular marrow and clusters of distributed plasma cells (A, original magnification $\times 400$, hematoxylin and eosin stain). Immunohistochemical staining was positive for CD38 $(B$, original magnification $\times 400)$ and CD138 $(C$, original magnification $\times 400)$. Kappa $(D$, original magnification $\times 400)$ and lambda light chain $(E$, original magnification $\times 400)$ in situ hybridization showed the monoclonal expression of the kappa light chain.
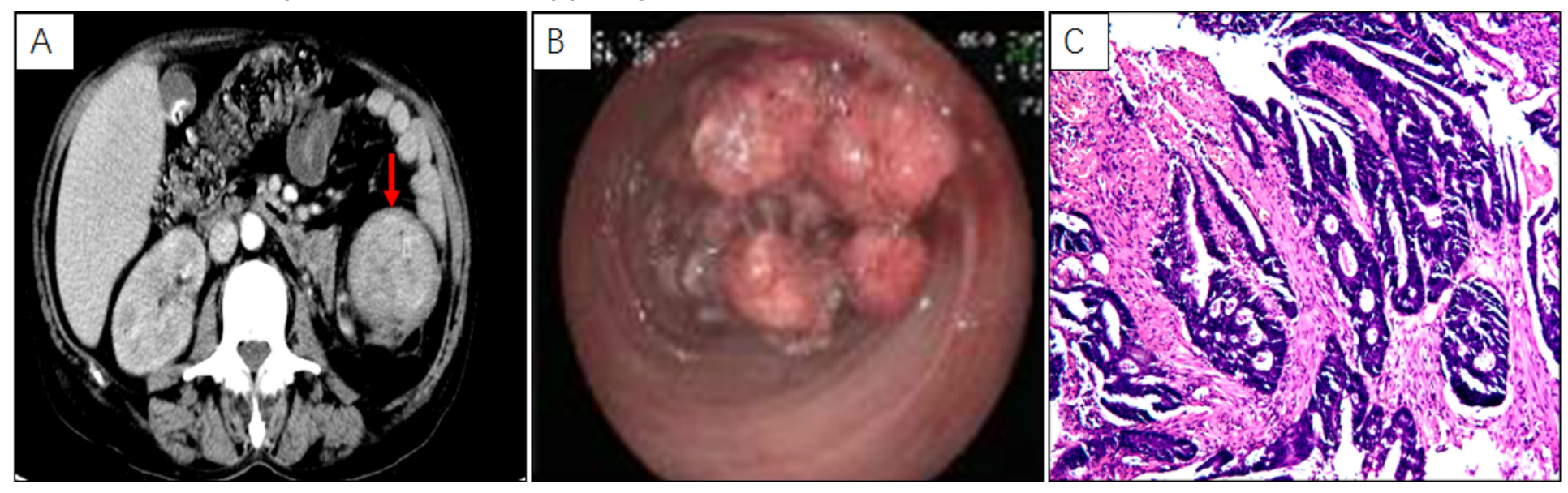


\section{Figure 3}

Abdominal CT showing marked thickening of the left intestinal wall and narrowing of the intestine (A, marked by a red arrow). Colonoscopy showed a cauliflower mass in the colon (B), and biopsy showed adenocarcinoma of the colon (C, original magnification $\times 200$, hematoxylin and eosin stain).

\section{Supplementary Files}

This is a list of supplementary files associated with this preprint. Click to download.

- CAREchecklistEnglish2013Finished.pdf 